\title{
Calibration of a High Resolution Airborne 3-D SAR
}

Dall, Jørgen; Grinder-Pedersen, Jan; Madsen, S.N.

Published in:

Proceedings of the International Geoscience and Remote Sensing Symposium

Link to article, DOI:

10.1109/IGARSS.1997.615329

Publication date:

1997

Document Version

Publisher's PDF, also known as Version of record

Link back to DTU Orbit

Citation (APA):

Dall, J., Grinder-Pedersen, J., \& Madsen, S. N. (1997). Calibration of a High Resolution Airborne 3-D SAR. In Proceedings of the International Geoscience and Remote Sensing Symposium (Vol. 2, pp. 1018-1021). IEEE. https://doi.org/10.1109/IGARSS.1997.615329

\section{General rights}

Copyright and moral rights for the publications made accessible in the public portal are retained by the authors and/or other copyright owners and it is a condition of accessing publications that users recognise and abide by the legal requirements associated with these rights.

- Users may download and print one copy of any publication from the public portal for the purpose of private study or research.

- You may not further distribute the material or use it for any profit-making activity or commercial gain

- You may freely distribute the URL identifying the publication in the public portal 


\title{
Calibration of a High Resolution Airborne 3-D SAR
}

\author{
J. Dall, J. Grinder-Pedersen, S. N. Madsen \\ Danish Center for Remote Sensing, Dept. of Electromagnetic Systems \\ Technical University of Denmark, B-348, DK-2800 Lyngby, Denmark \\ Phone: +45 4525 3800, Fax: +45 4593 1634, E-mail: jd@emi.dtu.dk
}

\begin{abstract}
The potential of across-track interferometric (XTI) synthetic aperture radar (SAR) for producing high resolution 3-D imagery has been demonstrated by several airborne systems including EMISAR ${ }^{1}$, the dual frequency, polarimetric, and interferometric SAR developed at the Dept. of Electromagnetic Systems (EMI). In order to achieve a high geodetic fidelity when using such systems operationally, calibration procedures must be applied. Inaccurate navigation data and system parameters as well as system imperfections must be accounted for. This paper presents theoretical models describing the impact of key error contributors on the measured terrain elevation. The models are used in estimating calibration parameters on the bases of distributed targets with known elevation. Finally, the calibration procedure is applied to EMISAR data collected by the Danish Center for Remote Sensing (DCRS ${ }^{2}$ ), and the stability of the estimated parameters is examined in order to assess the general utility of the procedure.
\end{abstract}

\section{INTRODUCTION}

Many airborne XTI systems are capable of producing 3-D imagery with height resolutions on the order of one meter, i.e. the stochastic height noise of these systems is about one meter. However, often systematic errors hamper the absolute accuracy of the generated digital elevation models (DEMs) unless multiple ground control points (GCPs) are used to correct the data. The need of such points complicates automation of the processing procedures, and it even prevents production of calibrated 3-D imagery in remote areas without GCPs.

The systematic errors can be divided into three different classes, viz. those originating from

- insufficient navigation system accuracy

- insufficient system parameter knowledge

- system imperfections

${ }^{1}$ Development of EMISAR has been supported by the Thomas B. Thriges Foundation, the Danish Technical Research Counsil (STVF), the Royal Danish Air Force (RDAF), the Technical University of Denmark, the Joint Research Centre (JRC) and by the Danish National Research Foundation.

${ }^{2}$ The Danish Center for Remote Sensing is finansed by the Danish National Research Foundation.
Navigation data of importance are the roll angle and the platform position which directly couples to the absolute pixel position. The system parameters include the channel delay, the channel phase shift including the phase ambiguity number, the baseline length and angle. The errors of the first two classes cause calibration errors that vary slowly and monotonously in the range direction. The system imperfections on the other hand give rise to errors of more complicated character. The imperfections addressed in this paper are multi-path propagation introduced by on-aircraft reflections and channel leakage [1]. Both cause oscillating phase errors and hence height errors that are generally not monotonous.

By setting up models relating the parameters of the error contributors to the systematic height errors, calibration parameters can be estimated from distributed targets with known elevation [1], e.g. a sea surface with waves giving the signal-to-noise ratio required to have sufficiently low height noise.

This calibration procedure has been applied to XTI data acquired with EMISAR in 1996. A total of 6 scenes from 3 different missions have been calibrated in order to examine the stability of the estimated parameters and to assess the general utility of the procedure.

In 1995 the L- and C-band polarimetric capabilities of EMISAR were supplemented with an XTI capability by adding two flush mounted C-band antennas in front of the wing. This antenna installation provides a baseline with a length of $\mathrm{B}=$ $1.14 \mathrm{~m}$ and an angle of $\alpha_{p}=34^{\circ}$ from the platform horizontal. The antennas are fully polarimetric, so either $\mathrm{HH}$ or VV polarized XTI data can be collected. Since the antennas are connected via the switch matrix also used for polarimetry the system can be operated in multiple XTI modes:

- single baseline (transmit on one antenna, receive on both);

- double baseline (sequentially transmit and receive on one antenna then transmit and receive on the other)

- dual baseline (sequentially transmit on one antenna and receive on both, then transmit on the other and receive on both, thus collecting both double and single baseline data).

EMISAR is flown on a Danish Air Force Gulfstream G-3 twin engine jet aircraft which is typically operated at $25.000 \mathrm{ft}$ when XTI data are collected. The navigation system includes INSs as well as a P-code GPS system. 


\section{MODELS}

Assuming that the Earth curvature can be neglected i.e. the SAR is at an altitude $\mathrm{H}$ above a flat reference plane, and that the baseline is much smaller than the slant range $R$, then the unwrapped interferometric phase is given by

$$
\phi=\phi_{1}-\phi_{2}=-\gamma \cdot \sin (\theta-\alpha), \gamma=2 \pi p B / \lambda
$$

where $\theta(>0)$ is the line-of-sight angle with respect to the nadir direction and $\alpha$ is the baseline angle. Thus $\theta-\alpha$ is the angle between the baseline normal and the line-of-sight direction, and $B \sin (\theta-\alpha)$ is the baseline component in the line-of-sight direction. $p=1$ in the single baseline mode and $p=2$ in the double baseline mode.

The terrain height $\mathrm{h}$ and the ground range $\mathrm{c}$ are given by

$$
\begin{aligned}
& h=H-R \cdot \cos (\theta) \\
& c=R \cdot \sin (\theta)
\end{aligned}
$$

The impact of navigation data errors is easily found from (1-3). The height sensitivity to a platform altitude error is trivial

$$
\frac{\partial h}{\partial H}=1
$$

and since the resulting baseline angle $\alpha$ equals the platform baseline angle $\alpha_{\mathrm{p}}$ plus/minus the roll angle $\alpha_{\mathrm{r}}$ (right/left looking SAR), the sensitivity to a platform roll error is

$$
\frac{\partial h}{\partial \alpha_{r}}=\left.\left.\frac{\partial h}{\partial \theta}\right|_{H, R} \frac{\partial \theta}{\partial \alpha}\right|_{\phi, B} \frac{\partial \alpha}{\partial \alpha_{r}}=R \cdot \sin (\theta) \cdot 1 \cdot( \pm 1)= \pm c
$$

The influence of erroneous system parameters is also found from Eqs. 1-3. The impact of an $\alpha_{p}$ error is the same as that of an $\alpha$ error (the opposite for a left-looking SAR). The sensitivities to a baseline length error and a phase error are

$$
\begin{aligned}
& \frac{\partial h}{\partial B}=\left.\left.\frac{\partial h}{\partial \theta}\right|_{H, R} \frac{\partial \theta}{\partial B}\right|_{\phi, \alpha}=R \cdot \sin (\theta) \frac{\tan (\theta-\alpha)}{B}=\frac{c \phi}{B \sqrt{\gamma^{2}-\phi^{2}}} \\
& \frac{\partial h}{\partial \phi}=\left.\left.\frac{\partial h}{\partial \theta}\right|_{H, R} \frac{\partial \theta}{\partial \phi}\right|_{B, \alpha}=R \cdot \sin (\theta) \frac{1}{-\gamma \cdot \cos (\theta-\alpha)}=\frac{-c}{\sqrt{\gamma^{2}-\phi^{2}}}
\end{aligned}
$$

The channel delay errors impacts the estimation of the absolute phase, which in turn impacts the unwrapped phase according to (7).

The remainder of this section addresses the third class of errors, those originating in system imperfections. Fig. 1 shows an on-aircraft reflection point located on the line connecting the two interferometry antennas. The signals propagating between an antenna and a target via this point is delayed and attenuated before it is added to the direct signal. By using the approximation $1+\mathrm{ae}^{\mathrm{j} \phi}=1+\mathrm{a} \cdot \cos \phi+\mathrm{ja} \cdot \sin \phi \approx \mathrm{e}^{\mathrm{ja} \cdot \sin \phi}$, where $\mathrm{a}<<1$ is the attenuation factor of the delayed signal, it can be shown that in all three operation modes the phase error caused by on-aircraft multi-path propagation can be expressed as

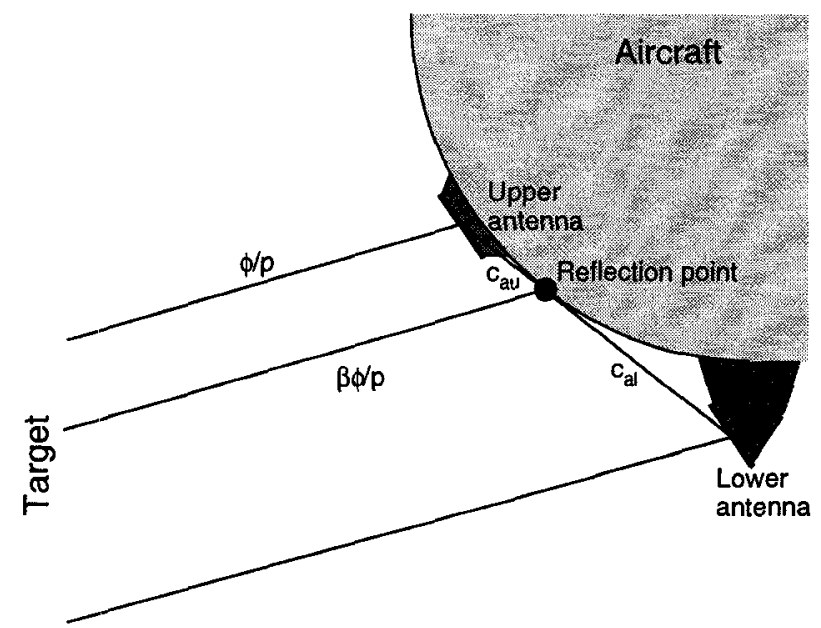

Fig. 1 On-aircraft multi-path. All propagation phases are oneway phases, and the phases to the target are differential with respect to the lower antenna. $c_{x}=a_{x} e^{j \phi_{x}}$.

$$
\phi_{A}=-p a_{l} \cdot \sin \left(\beta \cdot \phi / p+\phi_{a l}\right)-p a_{u} \sin \left((1-\beta) \cdot \phi / p-\phi_{a u}\right)
$$

$\beta B$ is the baseline from the lower antenna to the reflection point.

Fig. 2 outlines the switch matrix needed in XTI systems offering single, double, and dual baseline operation. The intended signal paths are indicate in black, the leakages in gray. In the figure the switch is set for transmission on the upper antenna. Note that the complex constants representing the leakages depend on the setting of the switch as indicated by the last subscript (upper transmit: $u$, lower transmit: 1). Using the above-mentioned approximation in combination with Fig. 2 it can be shown that the phase errors for double baseline, single baseline upper transmit, and single baseline lower transmit are

$$
\begin{aligned}
\phi_{d}= & -a_{e t u} \sin \left(\phi / 2+\phi_{o}+\phi_{t u}-\phi_{e t u}\right)-a_{e r u u} \sin \left(\phi / 2+\phi_{o}+\phi_{r u}-\phi_{e r u u}\right) \\
& -a_{e t l} \sin \left(\phi / 2+\phi_{o}-\phi_{t l}+\phi_{e t l}\right)-a_{e r l} \sin \left(\phi / 2+\phi_{o}-\phi_{r l}+\phi_{e r l l}\right)(9) \\
\phi_{u}= & -a_{e r u u} \sin \left(\phi+\phi_{o}+\phi_{r u}-\phi_{e r u u}\right)-a_{e r l u} \sin \left(\phi+\phi_{o}-\phi_{r l}+\phi_{e r l u}\right) \\
\phi_{l}= & -a_{e r u l} \sin \left(\phi+\phi_{o}+\phi_{r u}-\phi_{e r u l}\right)-a_{e r l} \sin \left(\phi+\phi_{o}-\phi_{r l}+\phi_{e r l l}\right)
\end{aligned}
$$

These three phase errors can be expressed in a generic form $\phi_{\mathrm{L}}=$ $a \cdot \sin (f \phi+o)$ which is used subsequently.
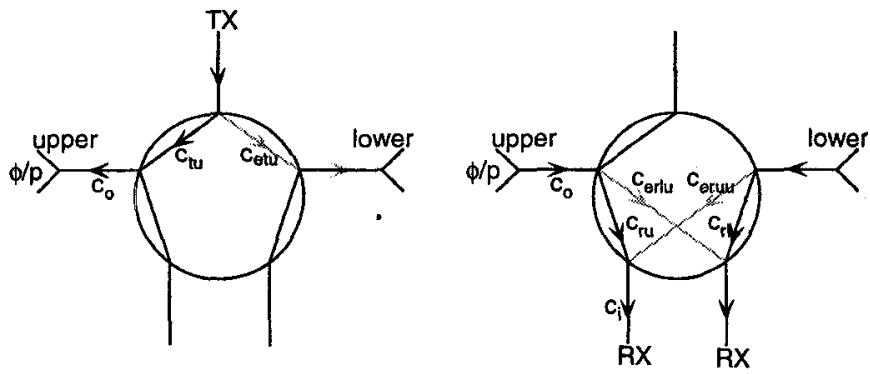

Fig. 2 Imperfect switch matrix. Left: transmit, right: receive. $c_{x}=a_{x} e^{j \phi_{x}}$ 


\section{CALIBRATION PROCEDURE}

According to Fig. 2 the measured phase is

$$
\phi_{m}=\phi+p \cdot \phi_{o}+(p-1) \cdot\left(\phi_{t u}-\phi_{t l}\right)+\left(\phi_{r u}-\phi_{r l}\right)+\phi_{i}+\phi_{a}+\phi_{L}
$$

The measured navigation and system parameters are: $\mathrm{H}_{\mathrm{m}}=$ $\mathrm{H}+\Delta \mathrm{H}, \quad \alpha_{\mathrm{rm}}=\alpha_{\mathrm{r}}+\Delta \alpha_{\mathrm{r}}, \quad \alpha_{\mathrm{pm}}=\alpha_{\mathrm{p}}+\Delta \alpha_{\mathrm{p}}$, and $\mathrm{B}_{\mathrm{m}}=\mathrm{B}+\Delta \mathrm{B}$. Using these parameters the measured height becomes

$$
h_{m}=h+\frac{\partial h}{\partial \phi}\left(\phi_{m}-\phi\right)+\frac{\partial h}{\partial H} \Delta H+\frac{\partial h}{\partial \alpha_{r}} \Delta \alpha_{r}+\frac{\partial h}{\partial \alpha_{p}} \Delta \alpha_{p}+\frac{\partial h}{\partial B} \Delta B
$$

First the calibration procedure interprets the difference between this height and the true height $\mathrm{h}=\mathrm{h}_{\text {ref }}$ of a reference surface as a phase error which according to (7) is given by

$$
\begin{array}{r}
\Delta \phi=\frac{\partial \phi}{\partial h}\left(h_{m}-h_{r e f}\right)=p \cdot \phi_{o}+(p-1) \cdot\left(\phi_{t u}-\phi_{t l}\right)+\left(\phi_{r u}-\phi_{r l}\right)+\phi_{i} \\
+\phi_{a}+\phi_{l}+\sqrt{\gamma^{2}-\phi^{2}}\left(\frac{\Delta H}{c} \pm \Delta \alpha_{r}-\Delta \alpha_{p}\right)-\phi \frac{\Delta B}{B}
\end{array}
$$

Note that $\Delta \phi \neq \phi_{\mathrm{m}}-\phi$ because $\Delta \phi$ also includes terms introduced when the impacts of the other system parameter errors and navigation data errors are interpreted as phase errors.

Next the calibration procedure plots $\Delta \phi$ as a function of $\phi_{\mathrm{m}}(\phi$ is not known). Unlike the first three phase terms in (14), which are constants, and the altitude, roll and baseline terms, which are slowly varying functions of $\phi$ (and hence $\phi_{m}$ ), the two multipath terms are very sensitive to the shift caused by substituting $\phi_{\mathrm{m}}$ for $\phi$. In this context it is worth noting that $\phi_{\mathrm{L}}\left(\phi_{\mathrm{m}}\right)$ does not depend on $\phi_{0}$, as $\phi_{L}$ according to Eqs. 9-11 is a function of $\phi+p \phi_{0}=\phi_{m}-(p-1)\left(\phi_{\mathrm{tu}}-\phi_{\mathrm{t}}\right)+\left(\phi_{\mathrm{ru}}-\phi_{\mathrm{r} 1}\right)+\phi_{\mathrm{i}}-\phi_{\mathrm{A}}-\phi_{\mathrm{L}}$.

Finally, the procedure estimates the calibration parameters by fitting the curve forms of the terms in (14) to $\Delta \phi\left(\phi_{m}\right)$. For a typical EMISAR geometry Fig. 3 plots these terms as functions of $\phi$ and the figure illustrates that the estimation is illconditioned as, for instance, a combined phase and roll error is fairly indistinguishable from an altitude error.

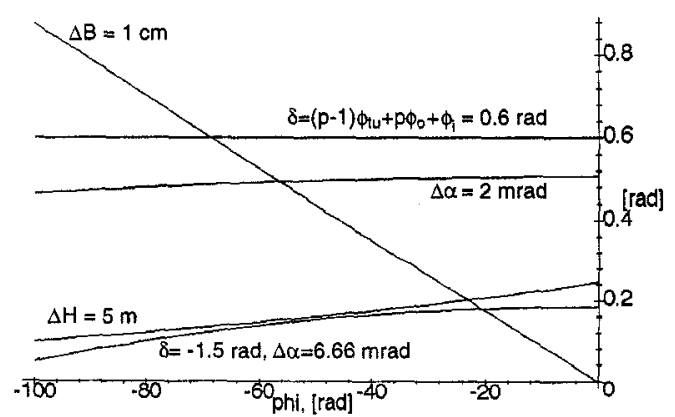

Fig. 3 Examples of $\Delta \phi$ contributors.

\section{EMISAR CALIBRATION}

This section shows some results based on six EMISAR data sets from three different flights, all collected over sea surfaces. The two interferometric channels are processed simultaneously and motion compensated to a common reference line, [2]. The processor also includes automatic determination of the absolute phase, [3]. The output products include co-registered amplitude data, height map, correlation map, and for calibration runs also absolute phase maps. The output data sets are orthorectified to a spherical $(s, c, h)$ coordinate system, [4]. Usually output products are generated at 5 or $10 \mathrm{~m}$ ground range pixel spacing.

The processing includes an amplitude, phase, and delay calibration based on signals from three internal calibration loops exercised just before and after every mapping. This internal calibration facility, which was originally intended for polarimetric calibration [5], covers the phase terms $c_{t u}$, and $c_{r u}$ but not $\phi_{0}$.

Fig. 4 shows $\Delta \phi\left(\phi_{\mathrm{m}}\right)$ for the six double baseline scenes after calibration of altitude, roll and phase. Baseline calibration was not included. The standard deviation of the roll and phase corrections are $1.8 \mathrm{mrad}$ and $0.3 \mathrm{rad}$, respectively. (The latter is $0.1 \mathrm{rd}$ when a single outlier is removed, while it is $0.5 \mathrm{rad}$ without internal calibration. The internal calibration in turn drifts less than $0.05 \mathrm{rad}$ from pre-map to post-map.) Since the figures correspond to height errors on the order of ten meters it is concluded that altitude, roll and phase must be calibrated on a scene by scene basis to achieve meter level accuracies. In Fig. 4 the slowly oscillating $\phi_{A}$ sinusoidal (first term, $\beta<0.5$ ) is superimposed the faster $\phi_{\mathrm{L}}$ sinusoidal. Table 1 lists the mean and standard deviation for the amplitude, frequency and offsets of these sinusoidals. The amplitudes correspond to height oscillations of a few meters and to an aircraft reflection suppression of $-20 \mathrm{~dB}$ and a switch isolation of -40 to $-30 \mathrm{~dB}$. The $\phi_{\mathrm{L}}$ frequency of 0.5 is consistent with (9), and the $\phi_{A}$ frequency of 0.11 corresponds to $\beta=0.23$ and hence a reflection point $\beta B=26 \mathrm{~cm}$ from one antenna. The offsets vary less than 2 percent of the sinusoidal periods and the amplitudes are also stable, so a fixed multi-path calibration can be applied to multiple scenes.

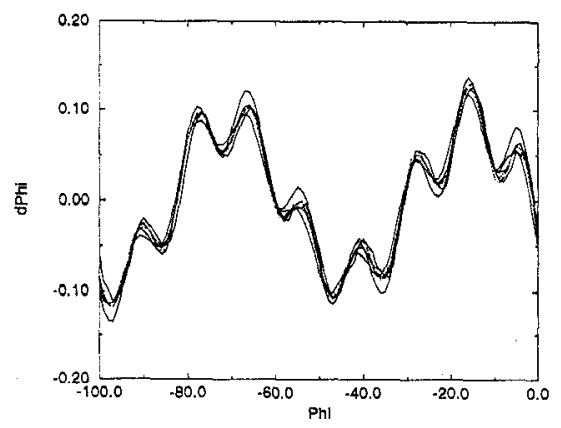

Fig. $4 \Delta \phi\left(\phi_{m}\right)$ after calibration of the navigation parameters and the system parameters. 
Table 1 Statistics for the multi-path phase terms.

\begin{tabular}{|l|c|c|c|}
\hline & $\begin{array}{c}\text { amplitude, a } \\
{[\mathrm{mrad}]}\end{array}$ & $\begin{array}{c}\text { frequency, } \mathrm{f} \\
{\left[\mathrm{rad}^{-1}\right]}\end{array}$ & $\begin{array}{c}\text { offset, o } \\
{[\mathrm{rad}]}\end{array}$ \\
\hline$\phi_{\mathrm{A}}(\beta<0.5$ term $)$ & $86 \pm 2$ & $0.11 \pm 0.00$ & $3.3 \pm 0.05$ \\
\hline$\phi_{\mathrm{L}}$ & $36 \pm 4$ & $0.50 \pm 0.00$ & $3.1 \pm 0.2$ \\
\hline
\end{tabular}

When the estimated sinusoidals are eliminated the second $\phi_{\mathrm{A}}$ term appears. It has a somewhat smaller amplitude and, as expected, a frequency corresponding to $1-\beta$.

One of the six data sets was collected in dual baseline mode. The frequencies of the sinusoidals extracted from the two single baseline products have proved to be twice that of the corresponding double baseline product, in accordance with Eqs. 8-11. Also, the amplitude of the $\phi_{\mathrm{A}}$ phase error differs by a factor of two is as expected from (8).

\section{CONCLUSION}

Accurate calibration is crucial for the generation of XTI products having not just a high resolution but also a high geodetic fidelity. Calibration based on ground control points is not easily automated and such points are rarely available in remote areas. This paper has presented a model-based calibration procedure using distributed targets with known elevation. The model includes navigation parameters, system parameters, and system imperfections. Errors from these three classes contribute to the EMISAR data, the calibration of which has been reported in this paper.
While the work with calibration procedures continues at DCRS, the EMISAR system is being upgraded in order to reduce the need of calibration. The navigation system, and in particular its attitude accuracy, will be improved, and a switch matrix with better isolation has been ordered.

\section{REFERENCES}

[1] S.N. Madsen, N. Skou, K. Woelders, and J. Granholm, "EMISAR Single pass Topographic SAR interferometer modes", International Geoscience and Remote Sensing Symposium, IGARSS'96, pp. 674-676, Lincoln, Nebraska, USA, May, 1996.

[3] David R. Stevens, Ian G. Cumming, and A. Lawrence Gray, "Options for Airborne Interferometric SAR Motion Compensation", IEEE Trans. GRS, vol. 33, pp. 409-420, March 1995.

[4] S.N. Madsen, "On absolute phase determination techniques in SAR interferometry", SPIE conference proceedings, Orlando, Florida, April 1995.

[5] Søren N. Madsen and Howard Zebker, "Imaging Radar Interferometry", Chapter 6 in Manual of Remote Sensing, Vol. III, 1997, in press.

[6] J. Dall, N Skou, E. Lintz Christensen, "Pulse-based Internal Calibration of Polarimetric SAR", IGARSS'94, 1994 International Geoscience and Remote Sensing Symposium, California, USA, August 1994. 